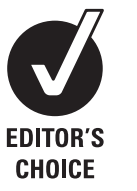

See Editorial, p 1723

1 Division of Cardiology, Severance Cardiovascular Hospital, Yonsei University Health System, Seoul, Republic of Korea

${ }^{2}$ Severance Biomedical Science Institute, Yonsei University Health System, Seoul, Republic of Korea

\section{Correspondence to}

Yangsoo Jang, Division of Cardiology, Severance Cardiovascular Hospital, Yonsei University Health System, 250 Seongsanno, Seodaemun-gu, Seoul 120-752, Republic of Korea; jangys1212@yuhs.ac

The first two authors contributed equally to the preparation of the manuscript.

Accepted 31 March 2011 Published Online First 20 May 2011

\title{
Impact of contrast-induced acute kidney injury with transient or persistent renal dysfunction on long-term outcomes of patients with acute myocardial infarction undergoing percutaneous coronary intervention
}

\author{
Jin Wi, ${ }^{1}$ Young-Guk Ko, ${ }^{1}$ Jung-Sun Kim, ${ }^{1}$ Byeong-Keuk Kim, ${ }^{1}$ Donghoon Choi, ${ }^{1}$ \\ Jong-Won Ha, ${ }^{1}$ Myeong-Ki Hong, ${ }^{1,2}$ Yangsoo Jang ${ }^{1,2}$
}

\begin{abstract}
Objective To investigate the long-term prognostic implications of contrast-induced acute kidney injury (Cl-AKI) with transient or persistent renal dysfunction in acute myocardial infarction (AMI) patients undergoing percutaneous coronary intervention (PCI).

Design A retrospective observational registry study.

Setting Clinical follow-up after PCI.
\end{abstract}

Patients and methods A total of 1041 PCl-treated AMI patients from the Infarction Prognosis Study registry. Cl-AKI was defined as an increase in serum creatinine $1>25 \%$ or $>0.5 \mathrm{mg} / \mathrm{dl}(>44.2 \mu \mathrm{mol} / \mathrm{l}))$ within 2 days after $\mathrm{PCl}$.

Main outcome measures Two-year cumulative event rate of all-cause death or renal failure requiring dialysis. Results Cl-AKI was observed in 148 patients (14.2\%). Patients with Cl-AKI had a higher rate of death or dialysis (25.4\% vs $6.3 \%, \mathrm{p}<0.001)$ at 2 years compared with patients without $\mathrm{Cl}-\mathrm{AKI}$. Cl-AKI was an important independent predictor of death or dialysis (HR 2.76, 95\% Cl 1.61 to $4.73, p<0.001)$ Persistent renal dysfunction after Cl-AKI was documented in 68 patients (45.9\%). Patients with transient renal dysfunction showed a lower 2-year event rate of death or dialysis compared with those with persistent renal dysfunction (17.9\% vs $34.1 \%, p=0.013)$; however, they showed a higher event rate compared with those without $\mathrm{Cl}-\mathrm{AKI}(17.9 \%$ vs $6.3 \%, \mathrm{p}<0.001)$.

Conclusion Transient and persistent renal dysfunction after $\mathrm{Cl}-\mathrm{AKI}$ was associated with increased short and longterm mortality and morbidity in AMI patients treated by $\mathrm{PCl}$. Better preventive strategies are needed to improve clinical outcomes in AMl patients at high risk of developing Cl-AKI.

Contrast-induced acute kidney injury (CI-AKI) is generally considered a reversible form of acute renal failure that begins soon after iodinated contrast administration during angiographic procedures. This hospital-acquired complication is of great concern because of its adverse effects on patients' clinical outcomes, including prolonged hospitalisation and increased morbidity and mortality. ${ }^{2} 2$ Important predisposing factors for CI-AKI include female gender, older age, diabetes mellitus, pre-existing renal insufficiency, advanced heart failure and intravascular volume depletion. ${ }^{3-5}$ The risk of CI-AKI is significantly higher among patients with acute myocardial infarction (AMI) undergoing percutaneous coronary intervention (PCI) than among the general population undergoing elective PCI. ${ }^{6-10}$ The most likely contributing factors for CI-AKI in patients with AMI are haemodynamic instability or impaired systemic perfusion caused by left ventricular dysfunction, large volume of contrast medium, or insufficient time to perform renal prophylactic therapies during contrast medium exposure. ${ }^{7}$ The purpose of this study was to determine the incidence of CI-AKI in patients with AMI undergoing PCI and to evaluate clinical predictors and long-term prognostic implications of CI-AKI in these patients.

\section{METHODS}

\section{Study population}

The registry of the Infarction Prognosis Study, a prospective single-centre cohort study, is maintained at the Severance Cardiovascular Hospital following an institutional review board-approved protocol. From the registry, we identified all consecutive patients 20 years of age or older who underwent PCI from May 2005 to July 2009. Patients exposed to contrast medium within 7 days before PCI or patients with end-stage renal disease requiring chronic dialysis treatment were excluded.

\section{Definitions}

CI-AKI was defined as a greater than $25 \%$ increase in serum creatinine level or greater than $0.5 \mathrm{mg} / \mathrm{dl}$ ( $>44.2 \mu \mathrm{mol} / \mathrm{l}$ ) serum creatinine within 2 days after intravascular administration of iodinated contrast medium when no other major kidney insult was identified. Recovery of renal function in CI-AKI patients was defined as return to serum creatinine level of $25 \%$ or less or $0.5 \mathrm{mg} / \mathrm{dl}$ or less $(\leq 44.2 \mu \mathrm{mol} / \mathrm{l})$ above the baseline level at $1 \mathrm{month}$ by a single measurement.

ST-segment elevation myocardial infarction was defined as characteristic chest pain with ST-segment elevation of $0.2 \mathrm{mV}$ or greater in two or more contiguous leads or new-onset left bundle branch block observed on electrocardiogram and positive troponin-T. Non-ST-segment elevation myocardial infarction was defined as myocardial infarction without ST-segment elevation. The Mehran score was calculated based on eight clinical and procedural variables such as age greater than 75 years, hypotension, congestive heart failure, intra-aortic balloon pump, serum creatinine, diabetes, anaemia and volume of contrast according to the previous report. ${ }^{5}$ 
The primary endpoint was defined as a composite of all-cause death and renal failure requiring dialysis. In addition, we also investigated outcomes in all-cause death as well as the composite event of death, dialysis and hospital admission due to cardiovascular events such as myocardial infarction, heart failure, stroke and target vessel revascularisation.

\section{Study protocol}

The serum creatinine concentration was routinely measured before PCI and $24 \mathrm{~h}, 48 \mathrm{~h}$ and 1 month after PCI. Creatinine clearance was calculated using the Cockcroft-Gault formula.

Patients received intravenous hydration with $0.9 \%$ normal saline ( $1 \mathrm{ml} / \mathrm{kg}$ per hour) for $12 \mathrm{~h}$ before and after PCI. However, for patients with ST-segment elevation myocardial infarction undergoing primary PCI, hydration was performed after PCI. In these patients prehydration was not generally performed because of limited time. The hydration rate was reduced to $0.5 \mathrm{ml} / \mathrm{kg}$ per hour in patients with left ventricular ejection fraction (LVEF) less than $40 \%$, overt heart failure or volume overload. Relevant baseline and follow-up laboratory and clinical data were recorded during the hospital stay. After hospital discharge, patients were clinically followed up at 1 month and every 3 months thereafter. If the patient did not attend a scheduled visit, outcome variables were obtained by telephone. The present study was approved by the hospital institutional review board and performed according to good clinical practice standards. Written informed consent was obtained from each patient before enrolment.

\section{Percutaneous coronary intervention}

PCI was performed with the femoral approach according to standard clinical practice. Patients received $250 \mathrm{mg}$ aspirin, $300 \mathrm{mg}$ clopidogrel (600 mg in case of ST-segment elevation myocardial infarction), and a bolus of $5000 \mathrm{U}$ heparin immediately after diagnosis of AMI, followed by an additional bolus of heparin during the procedure, if necessary. A non-ionic dimeric, iso-osmolality contrast medium, iodixanol (Visipaque, GE Healthcare, Princeton, New Jersey, USA), was used in 1033 patients $(99.7 \%)$. The other patients received a non-ionic monomeric, low-osmolar contrast medium (Ultravist, Bayer HealthCare Pharmaceuticals, Berlin, Germany). In general, target lesions were predilated with a balloon followed by stent implantation. Supportive pharmacological therapies, mechanical support, contrast medium dose and angioplasty technique were left to the discretion of the operator, according to our institute's clinical protocols and international guidelines. ${ }^{8}$

\section{Statistical analysis}

Continuous data are reported as mean $\pm \mathrm{SD}$, unless otherwise specified. Categorical data are expressed as absolute value and percentage. Continuous variables were compared by Student's $t$ test. Categorical variables were compared by $\chi^{2}$ or Fisher's exact test, as appropriate. A multivariate logistic regression model was used to determine independent risk factors of CI-AKI. The cumulative incidence of clinical events was estimated using the Kaplan-Meier method. The significance of the curves was tested using the log-rank test. Univariate and multivariate Cox regression analyses were performed to identify independent predictors of death and dialysis. Univariate analysis included variables such as age, gender, body mass index, smoking, diabetes mellitus, hypertension, dyslipidaemia, renal failure, past medical history, pattern of AMI (ST-segment elevation vs non-STsegment elevation myocardial infarction), shock, heart failure, multivessel disease, anaemia, primary PCI, contrast volume and medication. Variables with $\mathrm{p}<0.15$ in the univariate analysis were entered into the multivariate Cox regression analysis by forward stepwise selection. Statistical significance was defined as $\mathrm{p}<0.05$. Statistical analysis was performed by SPSS version 18.0.

\section{RESULTS}

\section{Patient characteristics}

Of the total of 1200 patients from the Infarction Prognosis Study registry cohort, 159 patients were excluded from analysis for the following reasons: PCI was not performed $(n=59)$, serum creatinine level was not properly checked after PCI $(n=76)$, or chronic dialysis was performed because of end-stage renal disease $(n=24)$. Therefore, 1041 patients (751 men $(71.9 \%)$, mean age $62.7 \pm 12.2$ years) were included in this study. Among them, ST-segment elevation myocardial infarction was diagnosed in $515(49.5 \%)$ patients and non-ST-segment elevation

Table 1 Baseline clinical characteristics of patients

\begin{tabular}{|c|c|c|c|}
\hline Variables & $\begin{array}{l}\text { CIN } \\
(n=148)\end{array}$ & $\begin{array}{l}\text { Non-CIN } \\
(n=893)\end{array}$ & p Value \\
\hline Men & $83(56.1 \%)$ & $665(74.5 \%)$ & $<0.001$ \\
\hline Age, years & $67.6 \pm 11.8$ & $61.8 \pm 12.1$ & $<0.001$ \\
\hline Age $>75$ years & $44(29.7 \%)$ & $127(14.2 \%)$ & $<0.001$ \\
\hline $\mathrm{BMI}, \mathrm{kg} / \mathrm{m}^{2}$ & $23.5 \pm 3.2$ & $24.2 \pm 3.5$ & 0.047 \\
\hline $\mathrm{BMI}<24 \mathrm{~kg} / \mathrm{m}^{2}$ & $92(62.2 \%)$ & $421(47.1 \%)$ & 0.002 \\
\hline Smokers & $48(32.4 \%)$ & $346(38.7 \%)$ & 0.170 \\
\hline Diabetes mellitus & $57(38.5 \%)$ & $212(23.7 \%)$ & $<0.001$ \\
\hline Hypertension & $85(57.4 \%)$ & $424(47.5 \%)$ & 0.023 \\
\hline Dyslipidaemia & $49(33.1 \%)$ & $348(39.0 \%)$ & 0.175 \\
\hline $\begin{array}{l}\text { Renal failure, } \mathrm{SCr}>1.5 \\
\mathrm{mg} / \mathrm{dl}(>132.6 \mu \mathrm{mol} / \mathrm{l})\end{array}$ & $31(20.9 \%)$ & $58(6.5 \%)$ & $<0.001$ \\
\hline Previous stroke & $9(6.1 \%)$ & $52(5.8 \%)$ & 0.899 \\
\hline Previous MI & $10(6.8 \%)$ & $49(5.5 \%)$ & 0.536 \\
\hline Previous PCI & $19(12.8 \%)$ & $118(13.2 \%)$ & 0.900 \\
\hline Previous CABG & $5(3.4 \%)$ & 17 (1.9\%) & 0.225 \\
\hline \multicolumn{4}{|l|}{ Clinical presentation } \\
\hline STEMI & $66(44.6 \%)$ & $449(50.3 \%)$ & 0.200 \\
\hline NSTEMI & $82(55.4 \%)$ & $444(49.7 \%)$ & 0.200 \\
\hline Cardiogenic shock & $37(25.0 \%)$ & $129(14.4 \%)$ & 0.001 \\
\hline Use of IABP & $17(11.5 \%)$ & $42(4.7 \%)$ & 0.001 \\
\hline Heart failure (LVEF <40\%) & $54(36.5 \%)$ & $160(17.9 \%)$ & $<0.001$ \\
\hline Mean LVEF, \% & $42.6 \pm 13.1$ & $49.3 \pm 12.4$ & $<0.001$ \\
\hline Multivessel disease & $101(68.2 \%)$ & $531(59.5 \%)$ & 0.043 \\
\hline Anaemia* & $49(33.1 \%)$ & $159(17.8 \%)$ & $<0.001$ \\
\hline Haemoglobin, g/dl & $13.1 \pm 2.3$ & $14.1 \pm 2.0$ & $<0.001$ \\
\hline Primary $\mathrm{PCl}$ & $55(37.2 \%)$ & $375(42.0 \%)$ & 0.269 \\
\hline Contrast volume, ml & $229 \pm 74$ & $217 \pm 72$ & 0.071 \\
\hline Contrast volume $>300 \mathrm{ml}$ & $39(26.4 \%)$ & $141(15.8 \%)$ & 0.002 \\
\hline \multicolumn{4}{|l|}{ Renal function } \\
\hline Pre-PCI SCr, mg/dl ( $\mu \mathrm{mol} / \mathrm{l})$ & $\begin{array}{l}1.25 \pm 0.76 \\
(110.5 \pm 67.2)\end{array}$ & $\begin{array}{l}1.03 \pm 0.31 \\
(91.1 \pm 27.4)\end{array}$ & 0.001 \\
\hline Pre-PCI CCr, ml/min & $59.3 \pm 30.1$ & $71.9 \pm 25.7$ & $<0.001$ \\
\hline Pre-PCI CCr $<60 \mathrm{ml} / \mathrm{min}$ & $85(57.4 \%)$ & $292(32.7 \%)$ & $<0.001$ \\
\hline $\begin{array}{l}\text { Post-PCl peak SCr, } \\
\mathrm{mg} / \mathrm{dl}(\mu \mathrm{mol} / \mathrm{l})\end{array}$ & $\begin{array}{l}1.91 \pm 1.23 \\
(168.8 \pm 108.7)\end{array}$ & $\begin{array}{l}1.04 \pm 0.31 \\
(91.9 \pm 27.4)\end{array}$ & $<0.001$ \\
\hline Mehran score & $9.4 \pm 6.8$ & $5.4 \pm 4.9$ & $<0.001$ \\
\hline \multicolumn{4}{|l|}{ Medication } \\
\hline Aspirin & $145(98.0 \%)$ & $866(97.0 \%)$ & 0.789 \\
\hline Thienopyridine & $127(85.8 \%)$ & $807(90.4 \%)$ & 0.091 \\
\hline Beta blocker & $112(75.7 \%)$ & $717(80.4 \%)$ & 0.197 \\
\hline ACE inhibitor/ARB & $118(79.7 \%)$ & $756(84.7 \%)$ & 0.130 \\
\hline Statin & $123(83.1 \%)$ & $787(88.1 \%)$ & 0.088 \\
\hline
\end{tabular}

*Defined as haemoglobin $<13 \mathrm{~g} / \mathrm{dl}$ for men and $<12 \mathrm{~g} / \mathrm{dl}$ for women.

$A R B$, angiotensin receptor blocker; BMI, body mass index; CABG, coronary artery bypass graft; $\mathrm{CCr}$, creatinine clearance; $\mathrm{CIN}$, contrast-induced nephropathy; IABP, intra-aortic balloon pump; LVEF, left ventricular ejection fraction; MI, myocardial infarction; NSTEMI, non-ST-segment elevation myocardial infarction; PCl, percutaneous coronary intervention; $\mathrm{SCr}$, serum creatinine; STEMI, ST-segment elevation myocardial infarction. 

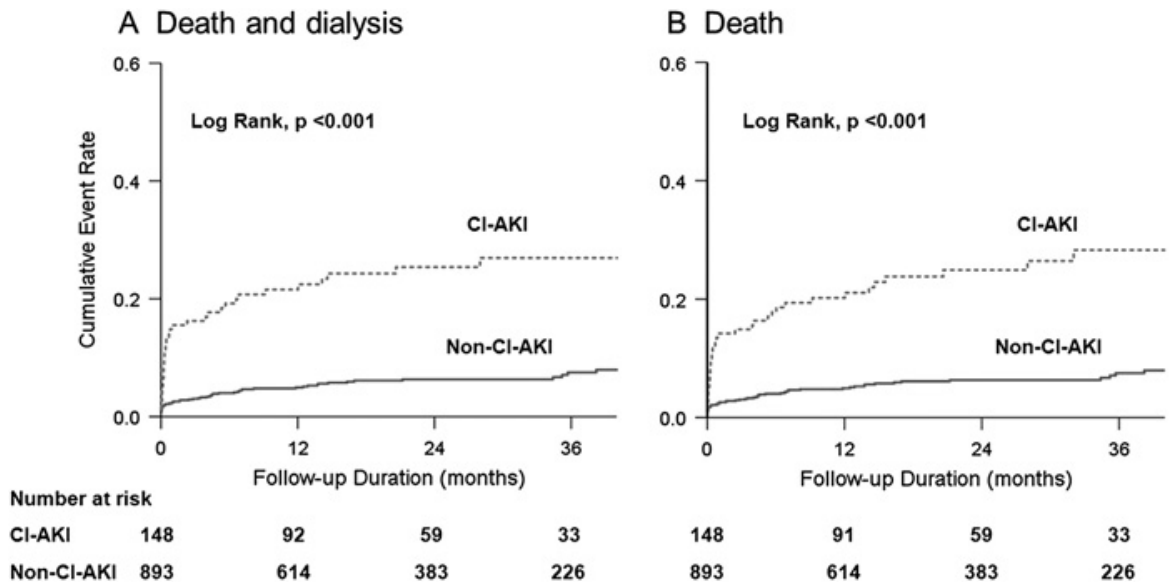

C Death, dialysis, and hospital admission

Figure 1 Cumulative event rates stratified by the presence or absence of contrast-induced acute kidney injury: (A) death or dialysis; (B) death; (C) death, dialysis, or hospital admission due to cardiovascular events. Cl-AKI, contrast-induced acute kidney injury.

myocardial infarction was diagnosed in 526 (50.5\%) patients. Overall CI-AKI was observed in $148(14.2 \%)$ patients. Table 1 shows the baseline clinical characteristics of patients with and without CI-AKI. There were notable significant clinical and procedural differences between subjects with and without CI-AKI. Patients with CI-AKI were older and more likely to be female. Certain comorbid conditions such as diabetes, hypertension and chronic renal failure were also observed more frequently in the CI-AKI group. Patients with CI-AKI exhibited more multivessel coronary artery disease and cardiogenic shock at initial presentation, and showed lower LVEF than patients without CI-AKI. Patients with CI-AKI had higher baseline serum creatinine levels $(1.25 \pm 0.76$ vs $1.03 \pm 0.31, p=0.001)$ and were more frequently exposed to a high contrast medium volume (>300 ml) during PCI (39 (26.4\%) vs 141 (15.8\%), $\mathrm{p}=0.002)$. The Mehran score was higher in the patients with CI-AKI than those without CI-AKI (9.4 \pm 6.8 vs $5.4 \pm 4.9, \mathrm{p}<0.001)$.

\section{Clinical outcomes: patients with CI-AKI versus patients without Cl-AKI}

Patients were clinically followed up for a mean duration of 22.8 \pm 15.9 months. Patients developing CI-AKI had a significantly longer hospital stay than patients without CI-AKI $(14.7 \pm 17.9$ vs $8.5 \pm 9.1$ days, $p<0.001)$, and the inhospital mortality rate was also markedly higher for patients developing CI-AKI (14.2\% vs $2.5 \%, \mathrm{p}<0.001)$. The 2 -year cumulative event rate of death or dialysis was higher in the CI-AKI group compared with the group without CI-AKI $(25.4 \%$ vs $6.3 \%$, log rank, $\mathrm{p}<0.001$ ), and the 2 -year mortality rate was $24.9 \%$ in the CI-AKI group versus $6.3 \%$ in the non-CI-AKI group (log rank, $p<0.001$ ) (figure 1). The 2-year cumulative rate of death, dialysis or hospital admission due to cardiovascular events was also higher in the CI-AKI group compared with the group without CI-AKI $(31.5 \%$ vs $16.7 \%$, log rank, $p<0.001)$. In the overall population, all-cause mortality occurred in 84 patients $(8.1 \%)$, re-infarction in $25(2.4 \%)$, target-vessel revascularisation in 31 $(3.0 \%)$, heart failure requiring hospital admission in 20 (1.9\%), cerebrovascular events in $11(1.1 \%)$ and renal failure requiring dialysis in five $(0.5 \%)$. The cause of death was cardiogenic in 52 patients $(61.9 \%)$, cerebrovascular in five $(5.9 \%)$, infection or malignancy in 15 (17.9\%) and unknown in 12 (14.3\%). Multivariate Cox regression analysis revealed that CI-AKI was an independent predictor of the rate of death or dialysis (HR 2.76, $95 \%$ CI 1.61 to $4.73, \mathrm{p}<0.001)$ after adjusting for confounding variables such as gender, age, baseline serum creatinine level,
LVEF and comorbidities (table 2). Elevated pre-PCI serum creatinine level $(>1.5 \mathrm{mg} / \mathrm{dl}, \mathrm{HR} 3.82,95 \%$ CI 2.12 to 6.90 , $\mathrm{p}<0.001$ ), previous stroke (HR 2.34, 95\% CI 1.20 to 4.57 , $\mathrm{p}=0.013$ ), body mass index less than $24 \mathrm{~kg} / \mathrm{m}^{2}$ (HR 1.99, 95\% CI 1.08 to $3.66, p=0.028)$, decreased LVEF ( $<40 \%$, HR $1.89,95 \% \mathrm{CI}$ 1.10 to $3.25, \mathrm{p}=0.021$ ) and anaemia (haemoglobinhaemoglobin $<13 \mathrm{~g} / \mathrm{dl}$ for men and $<12 \mathrm{~g} / \mathrm{dl}$ for women, HR $1.85,95 \% \mathrm{CI}$ 1.04 to $3.27, p=0.035$ ) were other independent predictors.

\section{Clinical outcomes: patients with transient versus persistent renal dysfunction}

Of 148 patients with CI-AKI, 80 patients (54.1\%) showed partial or complete recovery of renal function to the baseline level within 1 month, whereas impaired renal function after angiography remained unchanged in 68 patients (45.9\%). Baseline characteristics of the two subgroups did not differ significantly, except for hypertension, which was more frequently found in patients with persistent renal dysfunction ( $47.5 \%$ vs $69.1 \%, p=0.008)$. Overall, patients with persistent renal dysfunction after CI-AKI had a higher 2 -year death or dialysis rate $(34.1 \%$ vs $17.9 \%, p=0.013)$, a higher mortality rate $(34.6 \%$ vs $16.7 \%, p=0.012)$ and a higher death, dialysis, or hospital admission due to cardiovascular events rate $(42.1 \%$ vs $22.9 \%, p=0.021)$ than those with transient renal dysfunction (figure 2). However, in comparison with patients without CI-AKI, patients with transient renal dysfunction after

Table 2 Independent predictors of mortality or dialysis: multivariate cox regression analysis using forward stepwise selection

\begin{tabular}{|c|c|c|c|c|}
\hline & \multicolumn{2}{|l|}{ Univariate analysis } & \multicolumn{2}{|l|}{ Multivariate analysis } \\
\hline & HR (95\% Cl) & p Value & HR (95\% CI) & p Value \\
\hline $\begin{array}{l}\text { Pre-PCl } \\
\mathrm{SCr}>1.5 \mathrm{mg} / \mathrm{dl}\end{array}$ & 7.93 (5.17 to 12.15$)$ & $<0.001$ & $3.82(2.12$ to 6.90$)$ & $<0.001$ \\
\hline CIN & $4.39(2.88$ to 6.70$)$ & $<0.001$ & 2.76 (1.61 to 4.73$)$ & $<0.001$ \\
\hline Previous stroke & 2.51 (1.53 to 4.14$)$ & $<0.001$ & $2.34(1.20$ to 4.57$)$ & 0.013 \\
\hline $\mathrm{BMl}<24 \mathrm{~kg} / \mathrm{m}^{2}$ & $3.54(2.15$ to 5.85$)$ & $<0.001$ & 1.99 (1.08 to 3.66$)$ & 0.028 \\
\hline LVEF $<40 \%$ & $3.78(2.58$ to 5.55$)$ & $<0.001$ & $1.89(1.10$ to 3.25$)$ & 0.021 \\
\hline Anaemia* & 4.05 (2.87 to 5.70$)$ & $<0.001$ & 1.85 (1.04 to 3.27$)$ & 0.035 \\
\hline Age $>75$ years & 2.99 (2.09 to 4.26$)$ & $<0.001$ & & \\
\hline Female gender & 1.68 (1.18 to 2.38$)$ & 0.004 & & \\
\hline Diabetes mellitus & 1.56 (1.09 to 2.23$)$ & 0.016 & & \\
\hline Hypertension & 1.62 (1.14 to 2.29$)$ & 0.008 & & \\
\hline Shock & 3.47 (2.44 to 4.94$)$ & $<0.001$ & & \\
\hline
\end{tabular}

*Defined as haemoglobin $<13 \mathrm{~g} / \mathrm{dl}$ for men and $<12 \mathrm{~g} / \mathrm{dl}$ for women.

$\mathrm{BMI}$, body mass index; CIN, contrast-induced nephropathy; LVEF, left ventricular ejection fraction; $\mathrm{PCl}$, percutaneous coronary intervention; $\mathrm{SCr}$, serum creatinine level. 

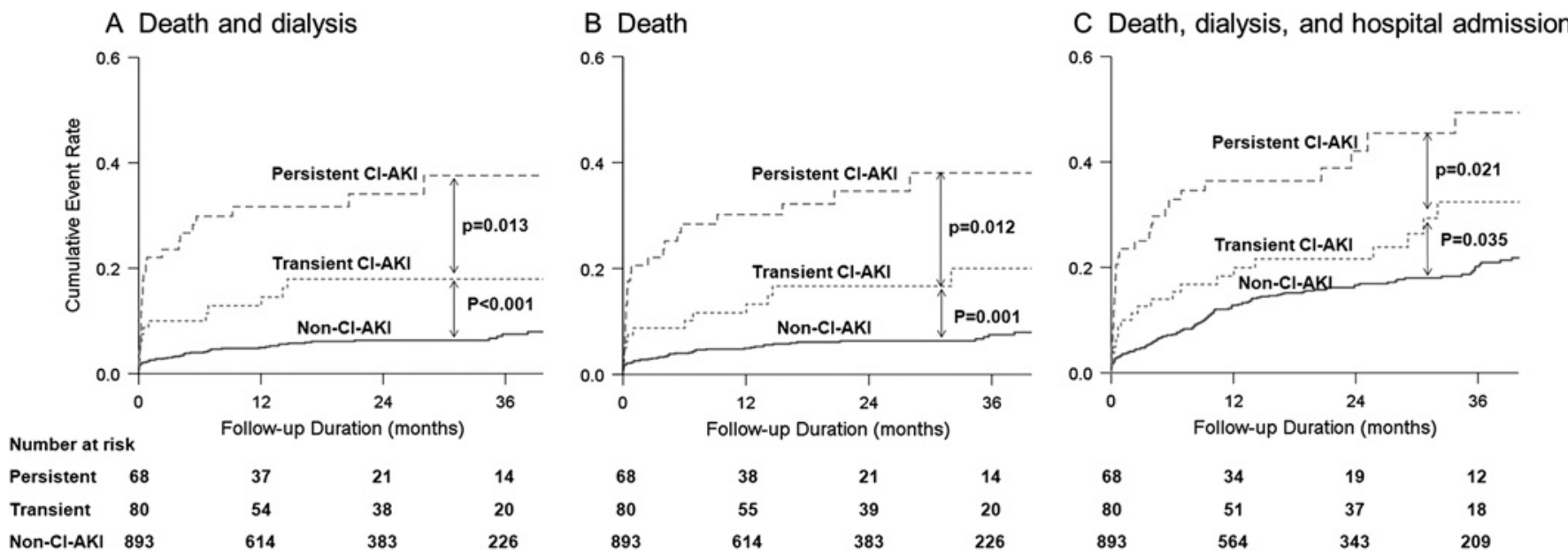

Figure 2 Cumulative event rates for patients with transient versus persistent renal dysfunction: $(A)$ death or dialysis; (B) death; (C) death, dialysis, or hospital admission due to cardiovascular events. CI-AKI, contrast-induced acute kidney injury.

CI-AKI still had a higher 2-year death or dialysis rate $(17.9 \%$ vs $6.3 \%, p<0.001)$, a higher mortality rate $(16.7 \%$ vs $6.3 \%, p=0.001)$ and a higher death, dialysis, or hospital admission due to cardiovascular events rate $(22.9 \%$ vs $16.7 \%, \mathrm{p}=0.035)$ than those with transient renal dysfunction.

\section{DISCUSSION}

The major findings of the present study were that patients with AMI who underwent PCI and subsequently developed CI-AKI had poorer short and long-term outcomes in the composite event of all-cause death and renal failure requiring dialysis than patients who did not develop CI-AKI. CI-AKI was an important independent predictor of the long-term event of mortality or dialysis in AMI patients treated with PCI. An additional finding of our study was that $45.9 \%$ of CI-AKI patients developed persistent renal functional impairment. Patients who experienced transient renal dysfunction showed better clinical outcomes than those with persistent renal dysfunction, but poorer outcomes than patients who did not develop CI-AKI.

CI-AKI is generally associated with increased short and longterm morbidity and mortality, although acute haemodialysis was uncommon except in patients with severe chronic kidney disease. ${ }^{1} 2411-13$ However, only a small number of studies have focused on the clinical implications of CI-AKI in AMI. Marenzi et $a l^{6}$ and Wickenbrock et al ${ }^{10}$ reported that CI-AKI was associated with a higher inhospital complication rate and mortality in patients with AMI undergoing PCI. In the present study, we found that CI-AKI was not only associated with short-term adverse outcomes, but also higher 2-year rates of mortality, dialysis or other cardiovascular events. The question of whether CI-AKI directly contributes to these adverse events is confounded by the findings of previous studies demonstrating that pre-existing renal insufficiency is associated with an increased risk of death in patients undergoing $\mathrm{PCI}{ }^{14}$ In our study, multivariate Cox regression analysis revealed that CI-AKI was an important independent predictor of death and dialysis after adjusting for confounding variables. Recently, Sgura et al ${ }^{15}$ reported that the Mehran score was a good predictor of the risk of developing major cardiovascular and cerebrovascular events in patients with ST-segment elevation myocardial infarction. The Mehran score was initially developed to predict CI-AKI after non-urgent PCI and includes eight clinical and procedural variables: age greater than 75 years; hypotension; congestive heart failure; intra-aortic balloon pump; serum creatinine; diabetes; anaemia and volume of contrast. ${ }^{5}$ Therefore, the development of CI-AKI in the acute phase of AMI may be considered a surrogate for more severe atherosclerosis and haemodynamic instability regardless of the underlying mechanism. ${ }^{15} 16$

In the present study, we also investigated whether the reversibility of renal function after the development of CI-AKI has any implication for clinical outcomes among AMI patients treated with PCI. CI-AKI is generally reversible and non-oliguric, with peak serum creatinine levels typically occurring at days 2 or 3 and returning to normal in most cases within 2 weeks. ${ }^{12} 17$ Persistent serum creatinine level elevation is considered to be rare $^{18} 19$ However, recent data from the Dartmouth Dynamic Registry showed that renal function of $54.6 \%$ of the patients with CI-AKI did not return to baseline after 2 weeks. ${ }^{20}$ Both transient and persistent postprocedural renal dysfunction were prognostically significant for mortality during extended followup in that study; however, the researchers observed no significant difference in the survival rate of patients with transient renal dysfunction caused by CI-AKI compared with those with persistent renal dysfunction.

In the present study, renal function was not restored to baseline at 1 month in $45.9 \%$ of patients who developed CI-AKI. In contrast with the previous study, CI-AKI patients with persistent renal functional impairment experienced worse short and long-term clinical outcomes than those with transient renal dysfunction. However, patients with transient renal dysfunction experienced more adverse clinical events than patients who did not develop CI-AKI. This may reflect that patients with persistent or transient renal dysfunction caused by CI-AKI have a different degree of atherosclerotic burden and haemodynamic instability. However, this could not be proved in our study. The clinical characteristics of the patients with persistent versus transient renal dysfunction were in general similar except for hypertension, which was more frequently observed in patients with persistent renal dysfunction.

CI-AKI often goes unnoticed by patients and physicians. Physicians tend to disregard mild or transient serum creatinine level elevation after coronary procedures that use contrast medium, especially when the serum creatinine level remains within the normal range or renal dysfunction improves rapidly. However, the results of our study indicate that CI-AKI unfavourably influences both short and long-term clinical outcomes in patients with AMI, even when renal function recovers rapidly. Once CI-AKI develops, treatment is limited to supportive 
measures while waiting for the renal impairment to resolve. Therefore, better preventive strategies and monitoring are needed to improve clinical outcomes in AMI patients at high risk of developing CI-AKI.

\section{Study limitations}

The present study had some limitations. First, our study is a post-hoc analysis of existing data. There was no adjudication process for clinical events. The need for dialysis or the use of hospital admission due to cardiovascular events as outcomes was limited by the unblinded nature of the data. Second, it was a moderate sized registry study in a single centre. Our findings need to be confirmed in a larger multicentre trial. Third, the incidence of CI-AKI in our study might have been underestimated because patients in whom creatinine was not adequately checked and patients with cardiogenic shock not surviving the first $48 \mathrm{~h}$ were excluded from the analysis. Fourth, the cause of renal function impairment after PCI (ischaemic, nephrotoxic, or atheroembolic) could not be determined precisely. Furthermore, because of methodological limitations inherent in retrospective registry analyses, our data cannot establish a definite aetiological link between worsening renal function after PCI and the increased risk of major adverse cardiorenal events and death. Finally, we were unable to determine whether patients had acute renal dysfunction, chronic kidney disease, or a combination of these two conditions. The serum creatinine level during hospitalisation may not reflect the steady state concentration in some patients with AMI and may be affected by non-renal factors such as hydration status.

Funding This study was partly supported by grants from the Korea Healthcare Technology R\&D Project, Ministry for Health, Welfare \& Family Affairs, Republic of Korea (nos A085012 and A102064), the Korea Health 21 R\&D Project, Ministry of Health \& Welfare, Republic of Korea (0412-CR02-0704-0001 and no A085136), and the Cardiovascular Research Center, Seoul, Republic of Korea.

Competing interests None declared.

Patient consent Obtained.

Ethics approval This study was conducted with the approval of the Institutional Review Board of Yonsei University Health System.

Provenance and peer review Not commissioned; externally peer reviewed.

\section{REFERENCES}

1. Best PJ, Lennon R, Ting HH, et al. The impact of renal insufficiency on clinical outcomes in patients undergoing percutaneous coronary interventions. J Am Coll Cardiol 2002;39:1113-19.
2. McCullough PA, Adam A, Becker CR, et al. Epidemiology and prognostic implications of contrast-induced nephropathy. Am J Cardiol 2006;98:5K-13K.

3. Parfrey PS, Griffiths SM, Barrett BJ, et al. Contrast material-induced renal failure in patients with diabetes mellitus, renal insufficiency, or both. A prospective controlled study. N Engl J Med 1989;320:143-9.

4. Rihal CS, Textor SC, Grill DE, et al. Incidence and prognostic importance of acute renal failure after percutaneous coronary intervention. Circulation 2002;105:2259-64

5. Mehran R, Aymong ED, Nikolsky E, et al. A simple risk score for prediction of contrast-induced nephropathy after percutaneous coronary intervention: development and initial validation. J Am Coll Cardiol 2004;44:1393-9.

6. Marenzi G, Lauri G, Assanelli E, et al. Contrast-induced nephropathy in patients undergoing primary angioplasty for acute myocardial infarction. J Am Coll Cardiol 2004;:44:1780-5

7. Marenzi G, Assanelli E, Marana I, et al. N-acetylcysteine and contrast-induced nephropathy in primary angioplasty. N Engl J Med 2006;354:2773-82.

8. Van de Werf F, Ardissino D, Betriu A, et al. Management of acute myocardial infarction in patients presenting with ST-segment elevation. The Task Force on the Management of Acute Myocardial Infarction of the European Society of Cardiology. Eur Heart J 2003;24:28-66.

9. Senoo T, Motohiro M, Kamihata $H$, et al. Contrast-induced nephropathy in patients undergoing emergency percutaneous coronary intervention for acute coronary syndrome. Am J Cardiol 2010:105:624-8.

10. Wickenbrock I, Perings C, Maagh P, et al. Contrast medium induced nephropathy in patients undergoing percutaneous coronary intervention for acute coronary syndrome: differences in STEMI and NSTEMI. Clin Res Cardiol 2009;98:765-72.

11. McCullough PA, Wolyn R, Rocher $L L$, et al. Acute renal failure after coronary intervention: incidence, risk factors, and relationship to mortality. Am J Med 1997; 103:368-75.

12. Gruberg L, Mintz GS, Mehran R, et al. The prognostic implications of further renal function deterioration within $48 \mathrm{~h}$ of interventional coronary procedures in patients with pre-existent chronic renal insufficiency. J Am Coll Cardiol 2000;36:1542-8.

13. Davidson CJ, Hlatky M, Morris KG, et al. Cardiovascular and renal toxicity of a nonionic radiographic contrast agent after cardiac catheterization. A prospective trial. Ann Intern Med 1989;110:119-24.

14. Reinecke H, Trey T, Matzkies F, et al. Grade of chronic renal failure, and acute and long-term outcome after percutaneous coronary interventions. Kidney Int 2003;63:696-701.

15. Sgura FA, Bertelli L, Monopoli D, et al. Mehran contrast-induced nephropathy risk score predicts short- and long-term clinical outcomes in patients with ST-elevationmyocardial infarction. Circ Cardiovasc Interv 2010;3:491-8.

16. Dangas G, lakovou I, Nikolsky E, et al. Contrast-induced nephropathy after percutaneous coronary interventions in relation to chronic kidney disease and hemodynamic variables. Am J Cardiol 2005;95:13-19.

17. Waybill MM, Waybill PN. Contrast media-induced nephrotoxicity: identification of patients at risk and algorithms for prevention. J Vasc Interv Radiol 2001;12:3-9.

18. Herts BR, Schneider E, Poggio ED, et al. Identifying outpatients with renal insufficiency before contrast-enhanced CT by using estimated glomerular filtration rates versus serum creatinine levels. Radiology 2008;248:106-13.

19. McCullough PA, Bertrand ME, Brinker JA, et al. A meta-analysis of the renal safety of isosmolar iodixanol compared with low-osmolar contrast media. J Am Coll Cardio 2006;48:692-9.

20. Brown JR, Malenka DJ, DeVries JT, et al. Transient and persistent renal dysfunction are predictors of survival after percutaneous coronary intervention: insights from the Dartmouth Dynamic Registry. Catheter Cardiovasc Interv 2008:72:347-54. 